\title{
MECHANICAL BEHAVIOUR OF HEALTHY AND DAMAGED HUMAN ARTERIES AND VALIDATION OF PARAMETERS DERIVED FROM EXPERIMENTS
}

Brigitta Krisztina Tóth, Imre Bojtár

Budapest University of Technology and Economics Department of Structural Mechanics brigitta.toth@mail.bme.bu

\begin{abstract}
Our research group aimed to develop a numerical model for the analysis of healthy and damaged human blood vessels (e.g. an aneurysm, an adverse vasodilatation) and to investigate the hyperelastic mechanical response of human brain arterial tissue. One of our tasks was to perform laboratory analysis on the specimens taken from the wall of the vessel, to calculate material model parameters for numerical models based on our measurements. Biaxial biomechanical characterization of living tissues - like the artery walls - provides important information about their in vivo behaviour. The aim of our research is to estimate stresses of the aneurysm wall and its parent artery, and to estimate the likelihood of a later aneurysm rupture, too. Laboratory measurements have been undertaken, we have taken the specimens of the vessel wall from human cerebral aneurysms and from control healthy artery carotis interna (both from surgery and cadavers), and then we investigated the biomechanical properties of samples in uniaxial and biaxial tensile tests. We attained for this by means of processing the values of the deformations and (Cauchy-)stresses arising from the wall. We verified the necessity of the parameters calculated from the laboratory measurements with finite element simulations performed on a real geometry aneurysm sack. From our study it may be concluded that there is a need for a constitutive model which describes the hyperelastic behavior of the human arterial wall. We demonstrated with numerical simulations that consideration for inhomogenity in investigations of diseased segments of blood vessels has crucial importance. We proved that damage taking place in aneurysm-sacks is many times more presumable than in healthy material parent arterial segments.
\end{abstract}

Keywords: hyperelastic material properties, internal carotid artery, aneurysm, biaxial measurement, finite element modelling, coupled modelling

\section{Introduction}

Since a few years we have been working in a research group, which aimed to develop a numerical model for the analysis of damaged human blood vessels, i.e. a model, which is hoped to once help medical doctors to judge whether a damaged section of the blood vessel (e.g. an aneurysm, an adverse vasodilatation) needs to be operated on, and if so, with what urgency.

Our task was to perform laboratory analysis on the specimens taken from the wall of the vessel, to calculate material model parameters for numerical models based on my measurements. We performed laboratory measurements on specimens of vessel wall in order to determine the strength properties of the vessel wall. Several members of staff at the Budapest University of Technology and Economics deal with the simulation of brain aneurysms (sack-like vasodilatations), which makes it important to learn the modified properties of such adverse vessel sections. Naturally, to make a comparison, for this purpose we have to know the material 
properties of the healthy vessels as check data. One of our aims is to determine material properties of both the adverse and the healthy vessels, which can be applied to computational models. Measurements on the material properties was necessary because we have not found any database regarding such vessels. It is the case because in several countries measurements on human tissues are prohibited by the law, and the scarce data collected with difficulty in the literature come from animal experiments.

During the numerical modelling we took into consideration the material properties of our own measurements to describe the elastic behaviour the vessel wall, therefore we can consider the material properties of the elastic tube in the coupled models.

\section{Methods}

Let us consider the mechanical material model which we can use for our intended mechanical analysis of the vessel wall. It is often assumed about polymer-like - live or lifeless - materials with inner microstructure that they do not change their elementary volume due to external mechanical influences, that is (in the case of homogeneous isotropic material) their bulk modulus is approximately infinite and their Poisson's ratio is 0.5 . The material of the vessel wall can be considered of this kind.

Stresses in hyperelastic, rubber-like materials are obtained by derivation of a deformation energy function assumed as known. We determined material properties for the so-called Mooney-Rivlin model, which is one of the most widely used model in numerical software packages. The Mooney-Rivlin energy function applies deformation invariants and the condition. The model has variants with two, three, five, or more parameters, too. Let us consider the energy function related to the variants with two, three, and five parameters without much detail. In the case of five parameters, the Mooney-Rivlin deformation energy function ${ }^{1}$ takes the form of

$$
\Psi=c_{1}\left(I_{1}^{\prime}-3\right)+c_{2}\left(I_{2}^{\prime}-3\right)+c_{3}\left(I_{1}^{\prime}-3\right)^{2}+c_{4}\left(I_{1}^{\prime}-3\right)\left(I_{2}^{\prime}-3\right)+c_{5}\left(I_{2}^{\prime}-3\right)^{2},
$$

where $I_{1}^{\prime}$ is the first deviatory deformation invariant, $I_{2}^{\prime}$ is the second deviatory deformation invariant, $c_{1}, c_{2}, c_{3}, c_{4}, c_{5}$ are parameters characterizing the deviatory deformation of the material. The three-parameter variant of the Mooney-Rivlin models differs from this only as it contains material constants $c_{1}, c_{2}, c_{4}$ in the equation above, while the two-parameter variant contains $c_{1}, c_{2}$ only.

It is essential for the numerical analysis of human artery walls to have at least an approximate knowledge on the material behaviour of the vessel wall. In order to reach this goal, laboratory measurements have been undertaken for several years in the Institute of Human Physiology and Clinical Experimental Research of the Semmelweis University. We joined this series of measurements, too. We have taken the specimens of the vessel wall from patients under operations, and from brain vessels extracted from cadavers (deceased patients), and then we have measured the stress-strain curves of the material of the vessel wall in one-dimensional and twodimensional tensile tests.

We cut $3 \mathrm{~mm}$ wide lengthwise and circumferential stripes out of the brain arteries (vessels of type internal carotid artery) obtained from cadavers for the purposes of the uniaxial tests and 8-by- 8 
$\mathrm{mm}$ large square-shaped specimens parallel to the above directions for the biaxial tests. The specimens were gradually pulled by a device equipped with strain gauges (fixed at both ends for uniaxial tests and fixed at all four sides for the biaxial tests), while the pulling force was digitally recorded (Figure 1).

During the processing of the measurement data, we have considered the original position of the specimens in the artery, basically in order to examine the inhomogeneous and anisotropic character of the material behaviour as accurately as possible.
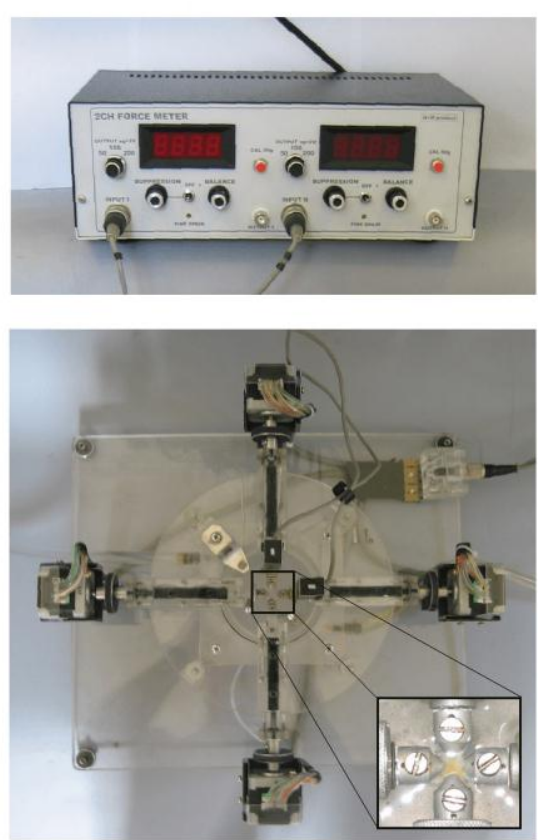

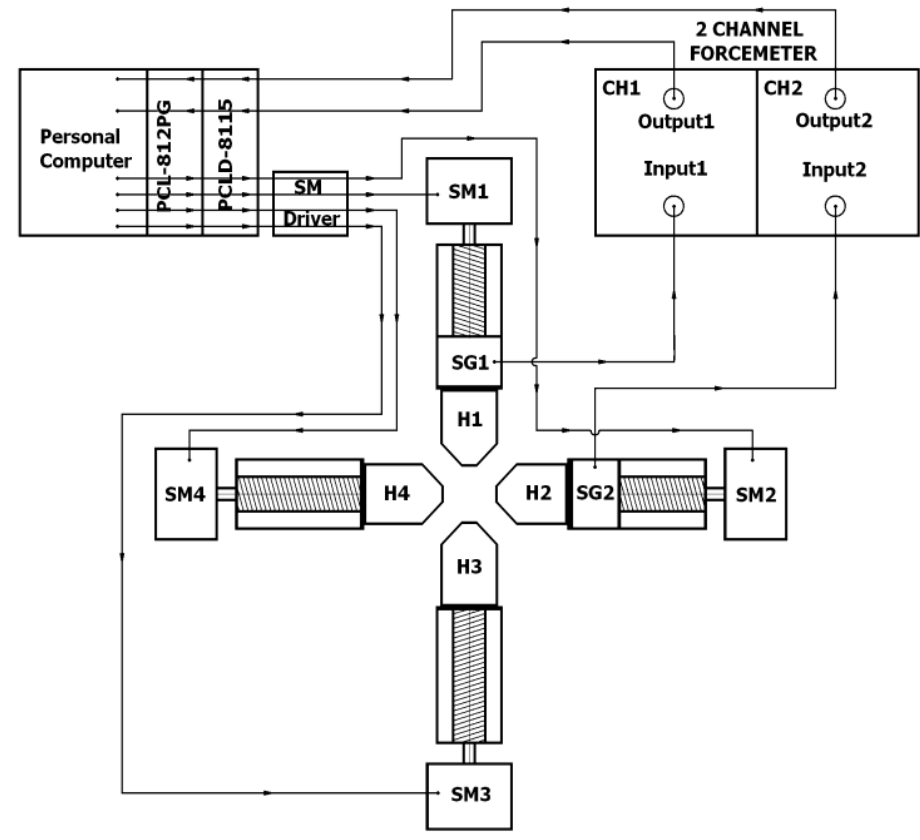

Figure 1. Biaxial laboratory measure device

(Institute of Human Physiology and Clinical Experimental Research, Semmelweis University)

The main parts of the biaxial device and the block chart are shown on the left and on the right, respectively. The specimen holders $(\mathrm{H})$ are displayed with the two strain gauge $(\mathrm{SG})$ attached. The two pairs of holders are placed perpendicular to each other. The output of the gauges is lead to a two-channel force measuring device, and the signals are transmitted to a PC by a multilab card. Displacements of the screws are actuated by 4 motors (SM), and the motions are transmitted to the PC by the multilab card and the SM control unit.

\section{Results and discussion}

It is a common property of the curves obtained that the initial line with small elastic modulus is followed by a steeper curve with an elastic modulus multiple of the initial one. Dividing the curves in two parts approximately linear sections are obtained, hence separate moduli of elasticity can be determined for each section. We have evaluated the measurements on the 12 specimens of internal carotid artery and determined the piecewise moduli of elasticity. During these measurements we have calculated moduli of elasticity of lengthwise, circumferential, and squareshaped (for the biaxial tests) specimens for all artery sections, therefore we was able to evaluate a total of 46 valid measurement data.

By means of the stress-strain curves calculated from my measurements on internal carotid 
arteries, we determined the hyperelastic Mooney-Rivlin material parameters required for the numerical analysis. By calculating the material properties we did not purely aim to provide data for our own research, but to make the foundations of a database which can be utilized by both medical doctors and engineers, and expanded in the future, too. Precisely for this purpose we have separately evaluated each specimen, and then tried to generalize the results. In the case of measurements on internal carotid artery used as a control group, we found the biaxial results particularly valuable. Plotting all curves obtained from biaxial measurements we can only deduce that a softer and a harder type of material can be distinguished. We fitted Mooney-Rivlin curves for all measurement series on internal carotid artery, and also on measurements we judged to be belonging to the softer and the harder types of material separately. For each measurement we considered the number of parameters $(2,3$, or 5$)$ in a way to get an acceptable approximation. The results are summarized in Table 1 without details, together with the averaged maximal stresses and strains related to the first damaged states, which are regarded as failure.

\begin{tabular}{|c|c|c|c|c|c|c|c|}
\hline & $\mathrm{c}_{1}[\mathrm{~Pa}]$ & $\mathrm{c}_{2}[\mathrm{~Pa}]$ & $\mathrm{c}_{3}[\mathrm{~Pa}]$ & $\mathrm{c}_{4}[\mathrm{~Pa}]$ & $\mathrm{c}_{5}[\mathrm{~Pa}]$ & Max. stress $[\mathrm{Pa}]$ & Max. strain $[\mathrm{m} / \mathrm{m}]$ \\
\hline ICA_all & 4248 & -3946 & & 22900 & & $1592000 \pm 679000$ & $0,8053 \pm 0,1650$ \\
\hline ICA_harder & 150900 & -136600 & & 75490 & & $2301000 \pm 1319000$ & $0,6258 \pm 0,1176$ \\
\hline ICA_softer & 35700 & -30560 & & 9998 & & $1000000 \pm 488800$ & $0,9550 \pm 0,2446$ \\
\hline
\end{tabular}

Table 1. Hyperelastic material model parameters obtained from averaging specimens of internal carotid artery (ICA), and the maximal stresses and strains related to the first irreversible damage.

Following our measurements on the walls of healthy arteries we dealt with the examination of aneurysms (adverse vasodilatations). As have mentioned before, we joined a series of measurements running for years at the Institute of Human Physiology and Clinical Experimental Research of the Semmelweis University. We took over the measurement series from Gábor Raffai, who performed measurements under the supervision of Emil Monos. ${ }^{2-4}$ He cut stripes out of the aneurysms parallel to the imaginary axis (meridional) and perpendicular ring-like direction (circumferential) for the uniaxial tests. We have performed the procedure shown for the internal carotid artery now for the total of 91 uniaxial measurement results, too. During this we determined stress-strain curves, and determined for each specimen whether the 3-parameter or the 5-parameter variant of the Mooney-Rivlin model approximates the diagrams more precisely. Since we had a significantly larger amount of measurement data were at our disposal, we examined differences with respect to gender and orientation. We formed a total of 9 groups: all together, males and females separately, circumferential (perpendicular to the main axis of the aneurysm) and meridional (approximately in the main axis of the aneurysm) separately, and also the cases of circumferential female, circumferential male, meridional female, and meridional male. Unfortunately, the data were insufficient to distinguish on the basis of pathological history. Distinctly visible difference is observable after averaging samples from women and men. We found that samples from men are harder: they get damaged by lower strains but higher stresses. This symptom remains, if we examine women and men belonging to the circumferential class, as well as (but less characteristically) we do the same in the meridional class. We did not show significant deviation between classes circumferential and meridional. In Table 2 we have summarized the Mooney-Rivlin material constants obtained by the grouping of aneurysms, 
together with the averaged maximal stresses and strains related to the first damage regarded as failure.

\begin{tabular}{|c|c|c|c|c|c|c|c|}
\hline & $\mathrm{c}_{1}[\mathrm{~Pa}]$ & $\mathrm{c}_{2}[\mathrm{~Pa}]$ & $\mathrm{c}_{3}[\mathrm{~Pa}]$ & $\mathrm{c}_{4}[\mathrm{~Pa}]$ & $\mathrm{c}_{5}[\mathrm{~Pa}]$ & Max. stress $[\mathrm{Pa}]$ & $\begin{array}{c}\text { Max. strain } \\
{[\mathrm{m} / \mathrm{m}]}\end{array}$ \\
\hline ANEURYSM all & -168300 & 187800 & & 196000 & & $431500 \pm 69420$ & $0,6834 \pm 0,0535$ \\
\hline ANEURYSM female & -101600 & 154200 & & 118900 & & $416200 \pm 68570$ & $0,6995 \pm 0,0767$ \\
\hline ANEURYSM male & -104200 & 148400 & & 205300 & & $524200 \pm 151300$ & $0,5928 \pm 0,1154$ \\
\hline
\end{tabular}

Table 2. Hyperelastic material model parameters of aneurysms - obtained by averaging and grouping, and the maximal stresses and strains related to the first irreversible damage.

Our next task was to create the model of the blood and the vessel wall with multiple couplings. On the one hand, the fluid (i.e. the blood) and the particles carried in it (red blood cells) mutually influence one another (see previous section, also in the case of the rigid wall), and on the other hand, due to the pulsing of the blood plasma, the motion of the vessel wall encircling the lumen of the blood reacts on the flowing field. Thus the so-called multiply coupled ('back and forth') modelling is doubly present in the problem we analyse. During the modelling the two ends of the artery section were fixed for brevity, and the tube was surrounded by elastic embedding representing brain tissues.

We carried out numerical analysis of blood flow in vessels in the range of internal carotid artery because this is the range of which we possess material properties. We determined these properties from the vessel models created using the averaged material properties of our biaxial tests (see the first row in Table 1). We performed fluid dynamics simulations on the fluid contained in the lumen of the vessel as before, and a solid mechanics finite element analysis on the encircling vessel, and the two were coupled. In each time step the output of the fluid dynamics simulation in the tube with rigid wall was applied as loads on the encircling vessel wall of hyperelastic material. The displacements arising in that tube were applied then to the fluid field in the next time step in such a way that the fluid space was modified with those displacements. The input fluid flow and the particles representing the red blood cells were physiological realistic (concentration of 40-50\%). We did not elaborate the effects of the elastic supports applied around the tube of the artery since this topic is investigated by another member of our department, Ferenc Nasztanovics.

Following the numerical simulations on the idealized geometry, we have performed simulations with finite element software package ANSYS 11.0 on real geometry as well - on a section of vessel containing a real brain aneurysm. The geometry and finite element mesh we used were obtained from Ádám Ugron, a member of the Department of Hydrodynamic Systems at BUTE, who deals with the simulations of fluid dynamical conditions of real brain aneurysms. Again in the numerical simulations we applied the hyperelastic material properties we gained from our laboratory measurements. We compared two cases. In the first case we applied the MooneyRivlin material parameters obtained by fitting a curve to the averaging of the healthy control arteries (internal carotid artery) to the entire vessel (including the aneurysm), see the first row in Table 1. In the second case the above mentioned parameters related to the general healthy arteries were exclusively applied to the parent arteries of the model and the aneurysm itself was modelled 
with Mooney-Rivlin material parameters obtained from the averaged curves of measurements on the aneurysms, see the first row in Table 2). Since geometric dimensions of this real model were in direct correspondence with our idealized tube with diameter of $3 \mathrm{~mm}$, the velocity of the blood was taken at a value of $0.35 \mathrm{~m} / \mathrm{s}$.

We found significant difference in stress values between the case of modelling the aneurysm with Mooney-Rivlin parameters of healthy vessels and the case of Mooney-Rivlin parameters of real aneurysms (see the von Mises stresses in Figure 2 and also the principal stresses in Table 3).

\begin{tabular}{|c|c|c|c|c|}
\hline & $\begin{array}{c}\text { von Mises } \\
{[\mathrm{Pa}]}\end{array}$ & $\begin{array}{c}\text { 1st pr. stress } \\
{[\mathrm{Pa}]}\end{array}$ & $\begin{array}{c}\text { 2nd pr. stress } \\
{[\mathrm{Pa}]}\end{array}$ & $\begin{array}{c}3 \text { rd pr. stress } \\
{[\mathrm{Pa}]}\end{array}$ \\
\hline $\begin{array}{c}\text { Dilatation as healthy arteria carotis } \\
\text { interna }\end{array}$ & 0,65 & 0,55 & 0,1 & $-0,4$ \\
\hline \begin{tabular}{c} 
Dilatation as aneurysm \\
\hline
\end{tabular} & 6 & 4 & 1,45 & $-2,4$ \\
\hline
\end{tabular}

Table 3. Maximal stresses in the aneurysm bag (pr.=principal)

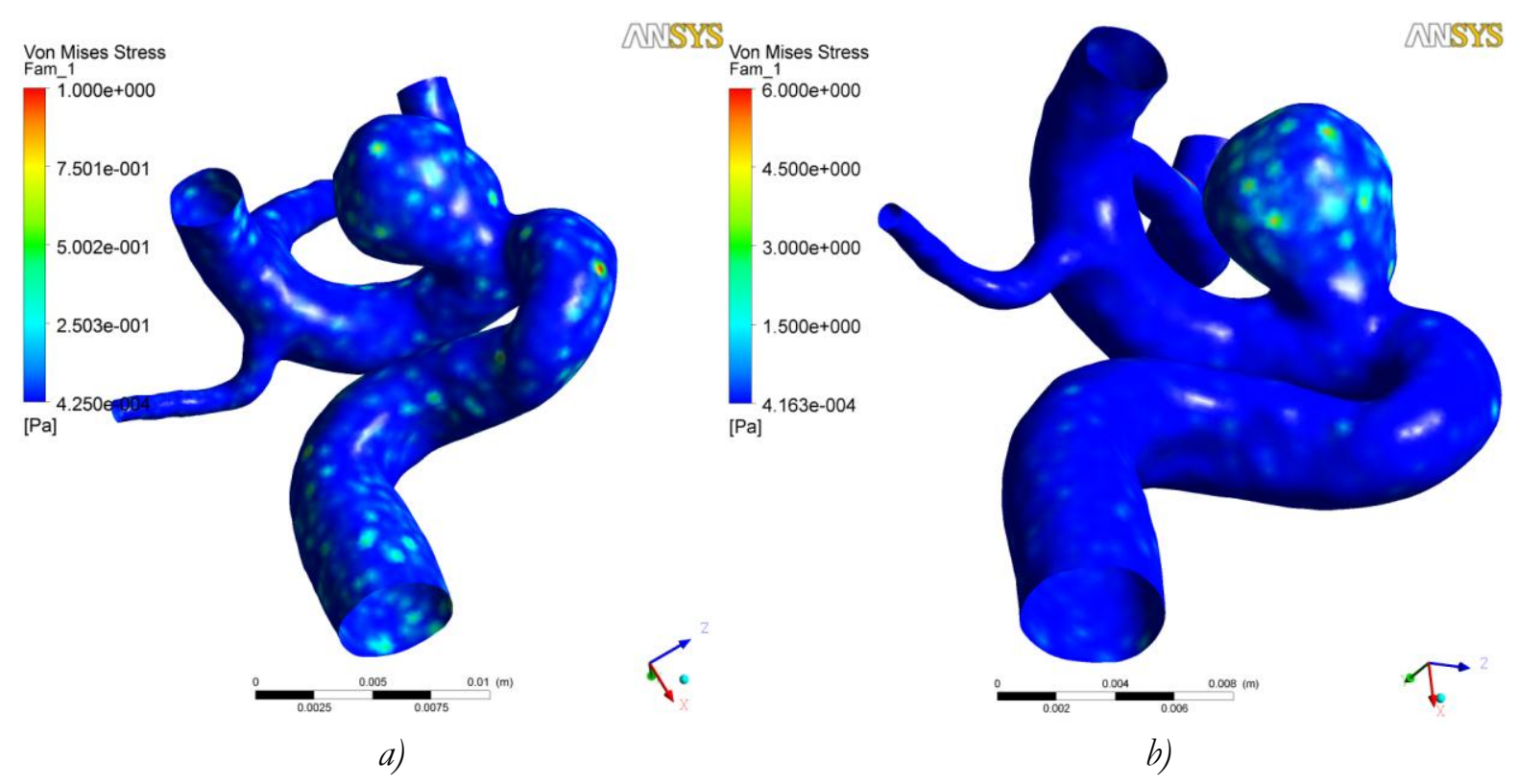

Figure 2. Von Mises stresses in an aneurysm with real geometry a) the entire vessel wall as internal carotid artery b) parent artery as internal carotid artery, and dilated section as aneurysm

\section{Conclusion}

We performed uniaxial and biaxial stretching measurements on carotid interna type arteries obtained from interventions and from cadavers, by means of which we determined the characteristic stress-strain curves of the artery wall resulting from stretching force. On the basis of the stress-strain curves we developed the bilinear elastic and the more parametric hyperelastic Mooney-Rivlin constitutive material model of the artery wall for the period before damage. We created the basis of a database for medical purposes by performing the statistical investigation of the received results. In the direction of cutting-out we did not show significant deviation. Based 
on the measurements we concluded that the type of artery under examination can be premordially characterized by a soft and a brittle material type. Our Mooney-Rivlin material parameters another data (maximal stresses and strains) obtained from arteries can be used as fundamental tools for further numerical examination of human arteries.

Based on the measurements we determined the characteristic stress-strain diagrams resulting from stretching forces of human cerebral aneurysms. Based upon these findings we developed the multi-parametric hyperelastic Mooney-Rivlin constitutive material model of this type of abnormal arterial wall related to the period before the damage. We created the basis of a medical database, and for each of the measurement data we determined whether the 3-parametric or the 5-parametric model is more suitable to describe. By performing the statistical investigation of the received results we established that characteristic divergences appear depending on the gender, however we did not find evident difference as a function of the cutting direction. We proved that the tissue samples obtained from males are more brittle in all sample categories: they deteriorate at shorter stretching and at higher values of stresses than the tissue samples of females.

We performed a numerical simulation of blood circulating in a real diseased arterial segment (including aneurysm) of dimension in the range of arteries of carotid interna type defined with Mooney-Rivlin material model with continuum based method by stationary flow. We proved that application of our material constants derived from the measurements performed on aneurysms yields significantly higher stresses compared to the application of material constants derived from the samples of healthy arteries of carotid interna type.

\section{REFERENCES}

1. Holzapfel GA, Gasser TC, Ogden RW. A new constitutive framework for arterial wall mechanics and a comparative study of material modes. Journal of Elasticity 2000; 61:1-48.

2. Monos, E. Hemodinamics, biomechanics of the blood circulation, Semmelweis Kiadó, Budapest, 43 pages, 2004

3. Tóth M, Nádasy GyL, Nyáry I, Kerényi T, Orosz M, Molnárka Gy, Monos E. Sterically inhomogeneous viscoelastic behavior of human saccular cerebral aneurysms. J.Vasc.Res. 1998; 35:345-55.

4. Orosz M, Molnárka Gy, Nádasy GL, Raffai G, Kozmann G, Monos E. Validity of viscoelastic models of vessel wall, 64th An. Meeting of the Hung. Physiol. Soc.; Budapest, Hungary, 1997

This work is connected to the scientific program of the "Development of quality-oriented and harmonized $R+D+I$ strategy and functional model at BME" project. This project is supported by the New Hungary Development Plan (Project ID: TÁMOP-4.2.1/B-09/1/KMR-2010-0002). 